\title{
1 Genome-wide analysis reveals signatures of 2 selection for gait traits in Yili horse
}

3 Ling-Ling Liu ${ }^{1}$, Chao Fang ${ }^{2}$, Jun Meng ${ }^{1}$, Johann Detilleux ${ }^{2}$, Wu-Jun Liu ${ }^{1,}$, Xin-Kui

$4 \mathrm{Yao}^{1, *}$

$5{ }^{1}$ Xinjiang Agricultural University, Urumqi, Xinjiang 830052, China;

$6 \quad{ }^{2}$ Department of Animal Production, Farah Research Centre from the Faculty of Veterinary

7 Medicine, University of Liège, Quartier Vallée 2,4000 Liège, Belgium

8

9

10

\section{Abstract}

High quality gaits play an important role in many breeding programs, but the genes of gait were limited at the moment. Here, we present an analysis of genomic selection signatures in 53 individuals from two breeds, using genotype data from the Affymetrix Equine 670K SNP genotyping array. The 11 selection regions of Yili horse were identified using an $F_{\mathrm{ST}}$ statistic and XP-EHH calculated in 200-kb windows across the genome. In total, 50 genes could be found in the 11 regions, and two candidate genes related to locomotory behavior (CLN6, FZD4). The genome of Yili horse and Russian horse were shaped by natural and artificial selection. Our results suggest that gait trait of Yili horse may related to two genes. This is the first time when whole genome array data is utilized to study genomic regions affecting gait in Yili horse breed.

Keywords: Yili horse, $F_{\mathrm{ST}}$, XP-EHH, Gait traits. 


\section{*Corresponding authors:}

Wu-Jun Liu, College of Animal Science, Xinjiang Agricultural University, Urumqi,

Xinjiang, Telephone number: 13079920452, E-mails: lwj_ws@163.com;

Xin-Kui Yao, College of Animal Science, Xinjiang Agricultural University, Urumqi,

Xinjiang, Telephone number: 13899805781,546462592@qq.com.

\section{Introduction}

Artificial selection in horse has resulted in divergent breeds that are specialized for either

sport or milk production or raised as dual-purpose breeds. Such selection strategies are

likely to have imposed selection pressures on particular regions of the genome that control

these traits. Such as the Arab horse was used to endurance races (Cervantes et al. 2009).

Gait is an important factor that affecting the pattern of horse locomotion. A few Chinese

horse breeds have a natural talent for gaits other than the common walk, trot and gallop, such as pace, which is a lateral two-beat gait (Hou 2012). Yili horse was called "tianma" and is a breeding breed in Xinjiang. This breed emerged about 2000 years ago in Western Han dynasty. In 1956, it was officially called "Yili horse" from the crossing of (Yang et al. 1987). Pace is a two-beat gait in which the horse moves the two legs on the same side of the body in a synchronized, lateral movement in contrast to the trot, where the diagonal front and hind legs move forward and backward together (Andersson et al. 2012). 
at high speed.

In recent years, methods for detecting selection signals using chip data have been widely used in livestock (Liu et al. 2018; Zhao et al. 2015; Ai et al. 2014; Gutiérrez et al. 2017). $F_{\mathrm{ST}}$ was used to detect the degree of differentiation between populations by the allele frequency (Wright 1950). XP-EHH was developed by Sabeti et al. on the basis of extended Haplotype Homozogyity (EHH) (Sabeti et al. 2007), which is efficiently to detect selected site. The companion study shows that selective signal method that based on haplotype-frequency was more accurate and reliable than method which based on single-site (Chen 2016). These two methods were widely used in domesticated animals, as in the cases of sheep (Yuan 2016; Zhu 2017), horse (Gu et al. 2009). However, the single $F_{\text {ST }}$ method may generate bias and false positives. Two methods would be more reliable if a reference population with a similar demographic history is available and if the allele under selection is close to fixation in one of the populations (Wagh et al. 2012). To minimize the bias and false positives, we combined these two approaches for the detection of selection. Genes related to gait traits are less reported, only the DMRT3 gene is described as the main gene involved in the determination of gait phenotypes in different horse breeds (Niina 2013).

The aim of this study was to identify selected regions related to gait trait of Xinjiang Yili horse breed based on Affymetrix Equine $670 \mathrm{~K}$ high-density chip using $F_{\mathrm{ST}}$ and XP-EHH.

\section{Materials and Methods}

\section{Animals}

A total of 53 horses from two populations were used in this study, including 40 Yili (YL) 

longitude $81^{\circ} 08^{\prime}$, latitude $\left.43^{\circ} 15^{\prime}\right)$. Jugular blood of $5 \mathrm{~mL}$ was collected from each animal in tubes treated by ACD anti-coagulation and stored at $-20^{\circ} \mathrm{C}$. The specimen was released.

\section{Quality control of SNPs}

DNA extraction and SNP genotyping were performed at the CapitalBio Technology in

Beijing. The Affymetrix equine SNP670 chip was used to genotype 53 horses. The SNP data was filtered by PLINK (v 1.9) software (Purcell et al. 2007). The following criteria

SNPs were filtered based on a minor allele frequency $(\mathrm{MAF})<0.03$. (3) SNPs were

filtered when there was no known autosomal genomic location, (4) SNPs were filtered

when the call rate $<0.9$, (5) SNPs were filtered that in Hardy-Weinberg equilibrium $(P<$ $\left.10^{-3}\right)$

Beagle ( $\mathrm{v}$ 4.0) was used to impute missing genotypes and infer the haplotype phase

(Browning et al. 2007).

\section{Population structure}

Principal component analysis (PCA) was performed using PLINK1.9 and the ggplot2 package in R (v3.4.4) was used to draw PCA figure (Wickham 2015). We removed SNPs in linkage disequilibrium in PLINK 1.9 with the command (--indep-pairwise 505 0.2).

\section{$F_{\text {ST }}$ test}

$F_{\text {ST }}$ was used to measure the distribution of genetic diversity among populations (Wright 1950; Weir et al. 1984), which uses the variance analysis to get unbiased estimates of 

of SNPs $\left(F_{\mathrm{ST}}>0.1699\right)$ as candidate sites.

\section{XP-EHH test}

For estimating XP-EHH, we use selscan (v1.1.0b) to confirm the selected sites detected by $F_{\mathrm{ST}}$ between RUL and YL populations (Szpiech et al. 2014; Pickrell et al. 2009), which is a more powerful method for selective sweeps that have above $80 \%$ frequency (Voight et al. 2006).

The top $0.5 \%$ of SNPs (XP-EHH > |-1.9405|) were selected as "candidate sites". RUL horse as the experimental population and YL horse as the control population.

\section{Bioinformatics analyses}

The $200 \mathrm{~kb}$ upstream and downstream of candidate sites were defined as the boundary of candidate regions. Horse annotated genes were searched via BioMart database of the ensemble genome browser (Arek 2011).

GO terms, including biological process, cellular component, and molecular function, and selected candidate regions, using the function annotation clustering tool from the Database for Annotation, Visualization an Integrated

Discovery

(DAVID v6.8, http://david.abcc.ncifcrf.gov/) (Huang et al. 2009). Corrections for multiple testing were performed by applying the Benjamini-Hochberg method (Benjamini et al. 1995), and GO terms and the KEGG pathways were considered signifcant at a $\mathrm{P}$ value lower than 0.05 . diagram of overlap sites of two methods. 


\section{Results}

\section{Genotype and quality control results}

113 After quality control, 143,652 SNPs were removed due to missing genotype data (--geno),

114 and 124,447 SNPs removed due to MAF, 23,702 SNPs on Un and X chromosome, 1,700

115 SNPs removed, which were in Hardy-Weinberg equilibrium $\left(P<10^{-3}\right)$. The remaining 116377,295 SNPs were used for subsequent analysis.

\section{Population stratification assessment}

118 Results of the PCA are shown in Figure 1. PC1 explained 3.13\% of variance, and PC2 explained $1.93 \%$ of variance. Result showed that RUL and YL horse were separated by PC1 and there was no mixed in two populations, which provided foundation for the subsequent selection of signal detection.

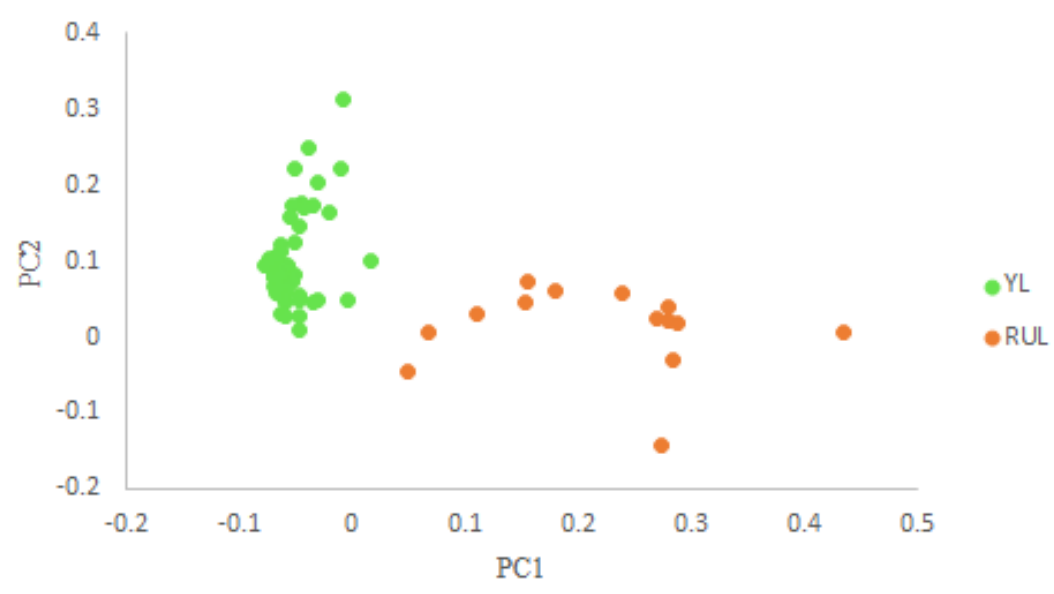

124 Figure1 Principal component analysis (PCA) of RUL and YL horse breeds 
A

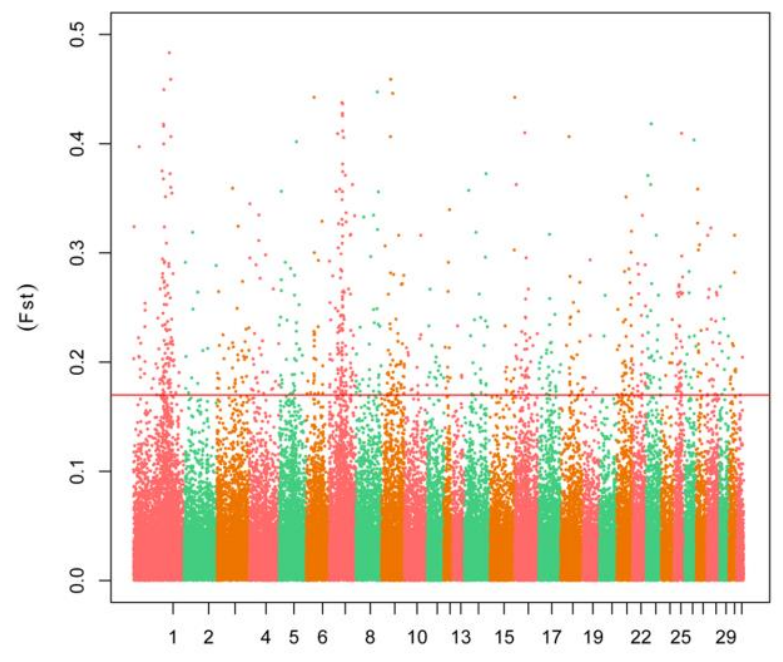

Chromosome
B

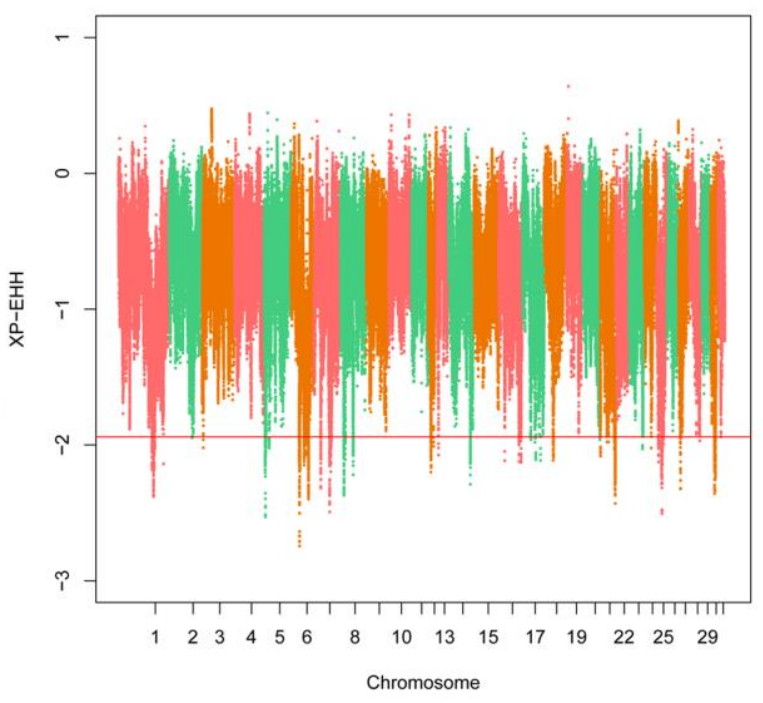

126

127

Figure 2 Selection signals of two population. (A) Genome-wide distribution of $F_{\mathrm{ST}}$ of

RUL-YL on autosomes. Note: the scale on the X-axis represents ID of chromosomes, the scale on the Y-axis is the $F_{\mathrm{ST}}$ value of each SNPs. The red solid line indicates value of top 0.5\%. (B) Genome-wide distribution of XP-EHH of RUL-YL on autosomes. Note: the scale on the X-axis represents ID of chromosomes, the scale on the Y-axis is the XP-EHH value of each SNPs. The red solid line indicates value of top $0.5 \%$.

\section{$F_{\text {ST }}$ test}

Vcftools was used to calculate $F_{\mathrm{ST}}$ of each SNPs between two populations. The $F_{\mathrm{ST}}$ value $\left(F_{\mathrm{ST}}=0.1699\right)$ was set as the threshold, and the highest $F_{\mathrm{ST}}$ of SNP $(\mathrm{AX}-103963716$, $\left.F_{\mathrm{ST}}=0.56\right)$ was located on chromosome 1. A total of 1,886 sites were selected. Manhattan map was showed in Figure 2.

\section{XP-EHH test}

The XP-EHH value (XP-EHH =-1.9405) was selected as the threshold. The SNP of largest XP-EHH value $(\mathrm{AX}-105009817, \mathrm{XP}-\mathrm{EHH}=-2.745)$ was located on chromosome 6. A total of 1,847 SNPs was selected as candidate sites, and the Manhattan plot was draw in Figure 


\section{Overlap analysis and gene annotation}

143 A total of 34 SNPs was detected by both methods (Figure 3), 11 overlapped regions containing 56 genes were annotated (Table 1).

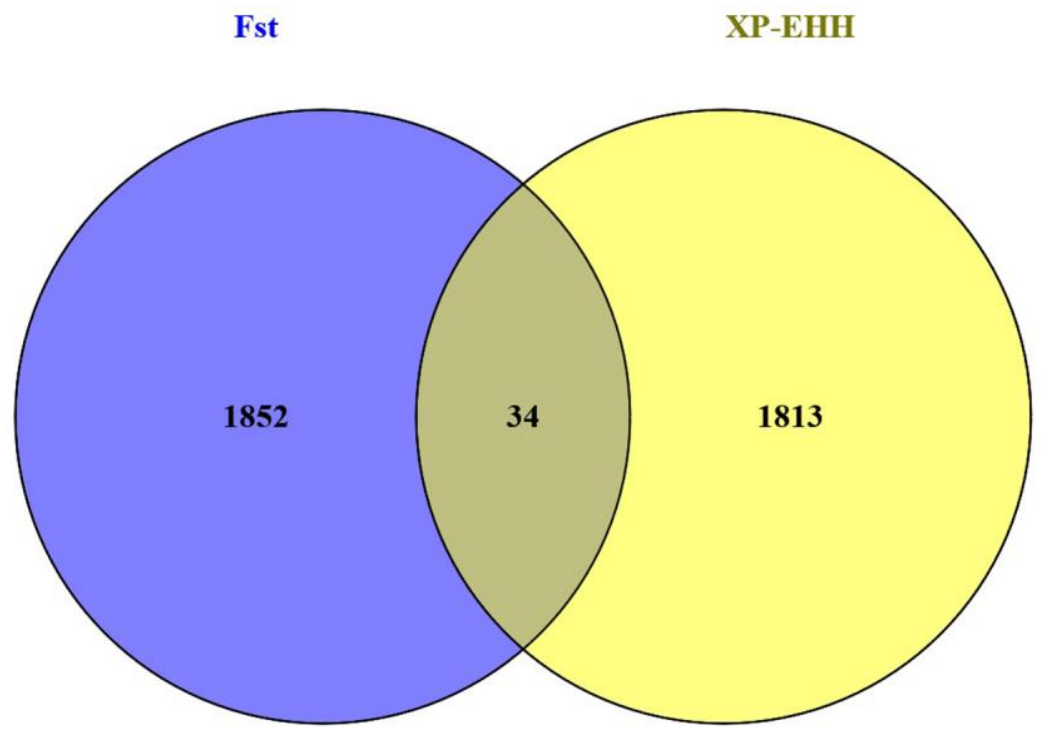

147 Figure 3 Venn diagram of two methods

148 Table 1 Selection regions and candidate genes of Yili horse population

\begin{tabular}{|c|c|c|c|c|c|}
\hline \multirow{2}{*}{ Chr } & Start & End & Max & Max & \multirow{2}{*}{ Candidate genes } \\
\hline & $(\mathrm{kb})$ & $(\mathrm{kb})$ & $F_{\mathrm{ST}}$ & XP-EHH & \\
\hline \multirow[t]{3}{*}{1} & 124.29 & 124.69 & 0.22 & 1.93 & ITGA11, FEM1B, CLN6, CALMLA, PIAS1 \\
\hline & & & & & $H E R C 1$, \\
\hline & & & & & ENSECAG00000015701,ENSECAG00000 \\
\hline \multirow[t]{3}{*}{1} & 128.43 & 128.83 & 0.18 & 1.98 & \\
\hline & & & & & 004063,ENSECAG00000016041,USP3,C \\
\hline & & & & & $A 12, A P H 1 B$ \\
\hline 5 & 5.80 & 6.40 & 0.19 & 2.07 & $B L Z F 1, C C D C 181$, \\
\hline
\end{tabular}


ENSECAG00000015067, SLC19A2,

ENSECAG00000006860, SELL, SELE, METTL18, C1orf112

PRMT8,SNORD112,ENSECAG00000021

038,ENSECAG00000021235,ENSECAGO

$\begin{array}{lllll}6 & 31.38 & 31.78 & 0.17 & 1.97\end{array}$

0000000564PARP11,ENSECAG00000012

642

TMEM135,FZD4,ENSECAG0000000605

$\begin{array}{lllll}7 & 58.68 & 59.08 & 0.19 & 2.00\end{array}$

$5, \operatorname{PRSS} 23$

ENSECAG00000011596,ENSECAG00000

$\begin{array}{lllll}7 & 61.46 & 61.86 & 0.17 & 2.06\end{array}$

006065,ENSECAG00000013426

FMNL2,ENSECAG00000001048,PRPF40

$\begin{array}{lllll}18 & 34.26 & 34.86 & 0.23 & 2.03\end{array}$

A,ARL6IP6,ENSECAG00000001190

$\begin{array}{llllll}21 & 55.11 & 55.51 & 0.21 & 2.14 & \text { N/A }\end{array}$

$\begin{array}{llllll}21 & 55.52 & 55.92 & 0.19 & 2.16 & \text { IRX2 }\end{array}$

$\begin{array}{llllll}21 & 55.93 & 56.33 & 0.18 & 2.11 & \text { IRX4 }\end{array}$

ERP44,INVS,TEX10,MSANTD3,TMEFF1,

CAVIN4,

$\begin{array}{lllll}25 & 6.67 & 7.47 & 0.19 & 2.02\end{array}$

ENSECAG00000007381,ENSECAG00000

025741,ENSECAG00000005825

149 Note: N/A indicated that this region was no gene. 
DAVID 6.8 was used to enrich selected genes. Finally, we identified five significantly enriched categories (Table 2), and one term was may be related to gait of Yili horse.

Table 2 Significantly enriched annotation clusters and functional terms

\begin{tabular}{|c|c|c|c|c|c|}
\hline Software & Term & Category & $\begin{array}{l}\text { Gene } \\
\text { count }\end{array}$ & $\mathrm{P}$-value & Benjamin \\
\hline \multirow[t]{10}{*}{ DAVID } & $\begin{array}{l}\text { Selectin } \\
\text { superfamily }\end{array}$ & INTERPRO & 3 & $6.1 \mathrm{e}-6$ & $4.9 \mathrm{e}-4$ \\
\hline & Domain:EGF-like & UP-SEQ-FEATURE & 5 & $6.8 \mathrm{e}-6$ & $1.1 \mathrm{e}-3$ \\
\hline & C-type lectin, & INTERPRO & 3 & $2.5 \mathrm{e}-4$ & $2.5 \mathrm{e}-3$ \\
\hline & conserved site & & & & \\
\hline & Locomotion & GOTERM_BP_DIRECT & 2 & $9.3 \mathrm{E}-3$ & $4.6 \mathrm{E}-2$ \\
\hline & involved & & & & \\
\hline & locomotory & & & & \\
\hline & behavior & & & & \\
\hline & oligosaccharide & GOTERM_MF_DIRECT & 2 & $9.2 \mathrm{E}-3$ & $3.8 \mathrm{E}-2$ \\
\hline & binding & & & & \\
\hline
\end{tabular}

Discussion

\section{Gait and Locomotion Performance}

Gait of horse is the pattern of movement of the limbs, it can be divided into Natural gaits and Ambling gaits according to the formation reasons. Natural gaits are defined as the innate and untaught gait, such as walk, trot, pace, canter, gallop and jump. Ambling gaits 
are obtained by training, such as fox trot, paso fino, racking, running walk, tölt and slow gaits (Ensminger 1969). Gaits are often characteristic of a particular breed (for a kinematic study see Nicodemus \& Clayton 2003) (Nicodemus et al. 2003), some horses are not able to learn a desired gait, others require extensive training, whereas some have a natural talent for ambling gaits.

Only DMRT3 gene is reported with horse gait. Anderson et al first show that a premature stop codon in the DMRT3 gene has a major effect on the pattern of locomotion in horses and find that Tölt is a unique ambling gait characteristic in Icelandic horse (Andersson et al. 2012). Subsequently, DMRT3 gene mutation was found to affect the Locomotion performance of horses in the Brazilian Mangalarga Marchador breed (Fonseca et al. 2017), the French trotter breed (Ricard 2015), American Saddlebred horses (Regatieri et al. 2016), Finn horses (Jäderkvist et al. 2015), Morgan and American Curly horses (Jäderkvist et al. 2014).

\section{Study on gait of china horse}

In china, there are lots of horse breeds, but studies on gait are rare. Only Han et al. (2015) tested genotype of DMRT3 gene from 14 Chinese local horse breeds, The DMRT3 nonsense mutation occurs mainly in Northwestern China, where horses having a natural talent for pacing. Yili horses show good performance in Locomotion, Yili horse inherited the good locomotion performance from its ancestor Kazakh horse. But its specific mechanism remains to be studied. At present, there are no reports on the study of the gait in Yili horse. Considering the influence of genetic background and natural conditions, there may be unique mechanisms in gait. In this study, we found other genes may influence gait. 
182

\section{Gene function}

In this study, one term was fund associated with locomotory behavior, and CLN6, FZD4 gene were fund in this term. We know that the doublesex and mab-3-related transcription factor 3 gene (DMRT3) encodes an important transcription factor involved in the coordination of the locomotor system controlling limb movement (Andersson et al. 2012), and that the CLN6 gene is one of eight genes related to the liposome of nerve cells, the CLN6 gene comprises seven exons and is predicted to encode a 311 amino-acid protein with seven hypothetical transmembrane domains (Gao et al. 2002; Wheeler et al. 2002). The CLN6 gene was recently identified, mutations in which cause one of the variant late infantile forms of NCL (vLINCL) (Teixeira et al. 2003). This finding demonstrates a role for CLN6 in gait.

FZD4 is known to be involved in the Wingless (WNT) signaling pathway, which plays an essential role in cardiac development (Gessert et al. 2010). But this gene was no report in gait. The results of this study need further verification.

\section{Conclusions}

In this study, we found eleven selected region, five enriched categories were found, one of them was associated with gait of Yili horse. The results of the present study indicate that the gait may be controlled by two genes (CLN6, FZD4) in Yili horse breed.

\section{Acknowledgements}

We thank Yue-Hui Ma and Lin Jiang at institute of animal science, Chinese academy of agricultural sciences, Beijing. At the same times, we thank Ming-Jun Liu and Wen-Rong Li at the Xinjiang academy of animal husbandry for assistance with analysis platform of 
biological information data. This project was supported by the National Natural Science

Foundation of China [Grant No.31560620]; National Sci-Tec R\&D project of China [Grant

No.2012BAD44B01].

\section{Ethics approval and consent to participate}

All experimental procedures involving animals were approved (animal protocol numbers:2017001) by the Animal Care and Use Committee of Xinjiang Agricultural University, Urumqi, Xinjiang, China.

\section{Literature Cited}

Andersson L. S., Larhammar M., Memic F., Wootz H., Schwochow D., et al., 2012

Mutations in dmrt3 affect locomotion in horses and spinal circuit function in mice. Nature

Ai H., Yang B., Li J., Xie X., Chen H., et al., 2014 Population history and genomic signatures for high-altitude adaptation in tibetan pigs. Bmc Genomics 15(1): 834. doi:

Arek K., 2011 BioMart: driving a paradigm change in biological data management.

Benjamini Y., Hochberg Y., 1995 Controlling the false discovery rate: a practical and powerful approach to multiple testing. J R Stat Soc Series B Methodol. 57(1): 289-300. Browning S.R., Browning B.L., 2007 Rapid and accurate haplotype phasing and missing-data inference for whole-genome association studies by use of localized haplotype clustering. American Journal of Human Genetics 81(5): 1084-1097. doi: 10.1086/521987 
analysis of morphofunctional traits in the Spanish Arab horse. Livestock Science 125(1):

43-49. doi: 10.1016/j.livsci.2009.03.006

Chen M.H., 2016 Selection signatures in the genomes of cattle and pigs. China

Agricultural University Beijing.

Danecek P., Auton A., Abecasis G., Albers C.A., Banks E., et al., 2011 The variant call

format and VCFtools. Bioinformatics

27(15):

2156-2158.

doi:

org/10.1093/bioinformatics/btr330

Ensminger M.E., 1969 Horses and horsemanship. Interstate Printers \& Publishers.

Fonseca M.G., Ferraz G.D.C., Pereira G.L., Curi R.A., 2017 A genome-wide association

study reveals differences in the genetic mechanism of control of the two gait patterns of the

Brazilian Mangalarga Marchador breedFrench trotter breed. Journal of Equine Veterinary

Science 53: 64-67. doi: 10.1016/j.jevs.2016.01.015

Gao H.L., Boustany R.M.N., Espinola J.A., Cotman S.L., Srinidhi L., et al., 2002

Mutations in a novel CLN6-encoded transmembrane protein cause variant neuronal ceroid

lipofuscinosis in man and mouse. American Journal of Human Genetics 70 (2): 324-335.

doi: $10.1086 / 338190$

Gessert S., Kühl M., 2010 The multiple phases and faces of Wnt signaling during cardiac

Circ Res. 107:186-199.

doi:

Gutiérrez-Gil B., Estebanblanco C., Wiener P., Chitneedi P.K., Suarezvega A., et al., 2017

High-resolution analysis of selection sweeps identified between fine-wool Merino and

coarse-wool Churra sheep breeds. Genetics Selection Evolution 49(1): 81. doi: 
249 Gu J., Orr N., Park S.D., Katz L.M., Sulimova G., et al., 2009 A genome scan for positive 250 selection in Thoroughbred horses. PLoS One 4(6): e5767. doi: 10.1371/journal.pone.0005767

Han H., Zeng L., Dang R., Lan X., Chen H., et al., 2015 The DMRT3 gene mutation in

Hou W.T., 2013 Modern Equine science. Chinese Agricultural Publishing House, Beijing,

Huang D.W., Sherman B.T., Lempicki R.A., 2009 Systematic and integrative analysis of large gene lists using DAVID bioinformatics resources. Nature Protocols 4:44-57.

Huang D.W., Sherman B.T., Lempicki R.A., 2009 Bioinformatics enrichment tools: paths toward the comprehensive functional analysis of large gene lists. Nucleic Acids Research 37: $1-13$.

Jäderkvist K., Johansson L., Mykkänen A., Mäenpää M., Andersson L.S., et al., 201536 The DMRT3 “Gait keeper” mutation affects harness racing performance and riding traits in Finn horses. Journal of Equine Veterinary Science 35 (5): 399. doi:

Jäderkvist K., Kangas N., Andersson L.S., 2014 Gaitedness is associated with the DMRT3

'Gait keeper' mutation in Morgan and American Curly horses. Animal Genetics 45 (6): 
271 Nicodemus M.C., Clayton H.M., 2003 Temporal variables of four beat, stepping gaits of

272 gaited horses. Applied Animal Behaviour Science 80(2): 133-142. doi:

Niina K., 2013 Association of the DMRT3 nonsense mutation with pattern of locomotion in five different horse breeds. Swedish University of Agricultural Sciences.

Pickrell J.K., Coop G., Novembre J., Kudaravalli S., Li J.Z., et al., 2009 Signals of recent positive selection in a worldwide sample of human populations. Genome Research 19(5): 826-837. doi: 10.1101/gr.087577.108

Purcell S., Neale B., Toddbrown K., Thomas L., Ferreira M.A.R., et al., 2007 PLINK: A tool set for whole-genome association and population-based linkage analyses. American Journal of Human Genetics 81(3): 559-575.

Regatieri I.C., Eberth J.E., Sarver F., Lear T.L., Bailey E., 2016 Comparison of DMRT3 genotypes among American Saddlebred horses with reference to gait. Animal genetics 47(5): 603-605. doi: 10.1111/age.12458

Ricard A., 2015 Does heterozygosity at the DMRT3 gene make French trotters better racers?. Genetics Selection Evolution 47(1): 1-16. doi: 10.1186/s12711-015-0095-7

Sabeti P.C., Varilly P., Fry B., 2007 Genome-wide detection and characterization of positive selection in human populations. Nature 449(7164): 913-918. 10.1038/nature06250 

mutations in the CLN6 gene causing a variant late infantile neuronal ceroid lipofuscinosis. Human Mutation 21(5):502-508. doi: 10.1002/humu.10207

Voight B.F., Kudaravalli S., Wen X., 2006 A map of recent positive selection in the human genome. PLoS Biology 4(3): e72. doi: 10.1177/1944451612464237

Wagh K., Bhatia A., Alexe G., Reddy A., Ravikumar V., et al., 2012 Lactase persistence and lipid pathway selection in the Maasai. PLoS One 7(9): e44751. doi: 10.1371/journal.pone.0044751

Weir B.S., Cockerham C.C., 1984 Estimating F-statistics for the analysis of population structure. Evolution 38(6): 1358-1370. doi: 10.2307/2408641

Wheeler R.B., Sharp J.D., Schultz R.A., Joslin J.M., Williams R.E., et al., 2002 The gene mutated in variant late-infantile Neuronal Ceroid Lipofuscinosis (CLN6) and in nclf mutant Mice encodes a novel predicted transmembrane protein. American Journal of Human Genetics 70 (2): 537-542. doi: 10.1086/338708

Wickham H., 2015 Ggplot2. Wiley Interdisciplinary Reviews Computational Statistics 3(2): 180-185.

Wright S., 1950 The genetical structure of populations. Nature 166(4215): 247-249. doi: 10.1111/j.1469-1809.1949.tb02451.x Yang Z., Xue Z.Y., Tang P.W., 1987 Analysis of the main factors of Yili horse breeding (in chinese). Journal of Animal Science and Veterinary Medicine 01:26-30. sheep. Chinese Academy of Agricultural Sciences Beijing. 
314 Zhao, F. , Mcparland, S. , Kearney, F. , Du, L. , \& Berry, D. P. . (2015). Detection 315 of selection signatures in dairy and beef cattle using high-density genomic 316 information. Genetics Selection Evolution, 47(1), 49.

317 Zhao F.P., Mcparland S., Kearney F., Du L., Berry D.P., 2015 Detection of selection

318 signatures in dairy and beef cattle using high-density genomic information. Genetics 319 Selection Evolution 47(1): 1-12. doi: 10.1186/s12711-015-0127-3

320 Zhu C.Y., 2017 Genome-wide association study, copy number variations and selection 321 signatures detect for sheep with different types of tails. China Agricultural University 322 Beijing. 